\title{
Ciclos de mejora docente en la asignatura de Química General basados en el trabajo guiado
}

\author{
JAVIER IGLESIAS SIGÜENZA \\ Universidad de Sevilla \\ Departamento de Química Orgánica \\ jiglesias@us.es \\ ORCID: https://orcid.org/0000-0001-8846-2303 \\ D.O.I.: http://dx.doi.org/10.12795/JDU.2018.i01.65 \\ Pp.: 1153-1169
}

\section{Resumen}

En esta comunicación se presentan una estrategia de mejora docente progresiva basada en los denominados Ciclos de Mejora Docente, los cuales se han realizado en una parte del temario de la asignatura "Química General" del Grado en Química.

Estos ciclos de mejora contemplan una reflexión sobre el modelo metodológico empleado, los contenidos fundamentales, la descripción de las distintas actividades realizadas, así como los resultados conseguidos tras la implantación de esta nueva forma de enseñanza, basada en un trabajo guiado a través de fichas docentes. Esta forma de enseñanza implica que el alumno vaya completando dichas fichas, manteniendo un razonamiento crítico a lo largo de todo el proceso de enseñanza-aprendizaje y descubriendo concepto a concepto todo lo necesario hasta llegar a alcanzar los objetivos. 
Para que esta forma de aprendizaje tenga éxito se requiere de un grado de compromiso alto y de una participación activa por parte del alumnado, ya que el conocimiento lo adquirirán a través de su propio trabajo, aunque éste sea guiado por el profesor.

Para planificar y abordar esta idea se recurrirá inicialmente a la realización del mapa de los contenidos prioritarios del tema, al desarrollo de las actividades, a la resolución de preguntas/problemas mediante debates en clase, así como al análisis e interpretación de los resultados obtenidos tras el ciclo de mejora.

Palabras claves: Química General, Grado en Química, Docencia universitaria, Experimentación docente universitaria, Fichas de trabajo guiado.

\section{Descripción del contexto de la intervención}

El ciclo de mejora docente se ha llevado a cabo en un tema correspondiente a una parte de la asignatura "Química General", asignatura anual de 18 créditos que se imparte en el primer curso del Grado en Química, siendo troncal o de formación básica en dicha titulación. Esta materia se imparte a razón de 4 horas teóricas semanales, y 2 horas prácticas (ejercicios y problemas) cada dos semanas, en grupos reducidos. En el presente curso académico hay un total de 68 alumnos matriculados en el grupo B, grupo del que soy responsable junto a otro profesor $(50 \%$ de la docencia cada uno). Las clases teóricas se imparten en un aula "ascendente" en la Facultad de Química de la Universidad de Sevilla dotada de una pizarra, ordenador y video-proyector. Para la impartición de las clases prácticas se divide al grupo en 4 partes (cada uno va un día de la semana) y la actividades se realizan en un seminario de la misma Facultad, dotado del mismo material. El ambiente de trabajo es bastante bueno entre ellos, así como la relación con el profesor, siendo la mayoría de los alumnos responsables, comprometidos con el proceso y dispuestos a aprender. 


\section{Diseño previo del Ciclo de Mejora Docente}

El modelo metodológico que había empleado hasta el momento para el desarrollo de esta asignatura había sido el de clases magistrales, condicionado por la forma de impartición de clases de mis compañeros y de la imposición de una lista de contenidos para explicar. Aunque las clases eran participativas, puesto que se intentaba interaccionar bastante con los alumnos, éstos acababan desconectando y se perdían en el transcurso de las mismas.

En este nuevo curso me he propuesto modificar la forma de explicar algunos temas, e ir abandonando la tradicional clase magistral (Finkel, 2008). De los distintos bloques, he elegido la lección más compleja, tediosa y abstracta del temario, la cual los alumnos deciden abandonar y excluir de su estudio a la hora de prepararse el examen final de la asignatura. Este cambio comenzaba con un replanteamiento de los conceptos fundamentales que el alumno debería tener, por lo que era necesario seleccionar y priorizar los contenidos (Figura 1), focalizándonos en lo esencial del tema.

Jornadas de Formación e Innovación Docente del Profesorado | № 1 (2018) Esta obra se distribuye con la licencia Creative Commons 


\section{1}

\section{Ecuación de onda}

Función de onda

sencillo? ¿Dónde se puede

colocar su único electrón?

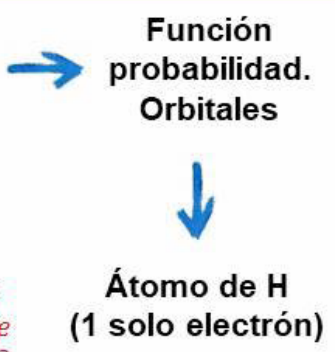

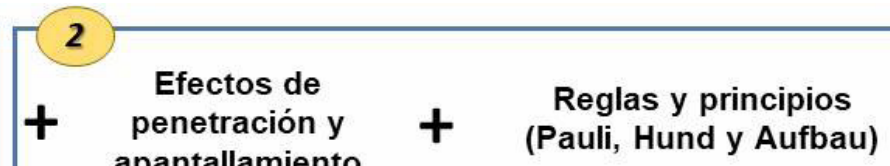
apantallamiento

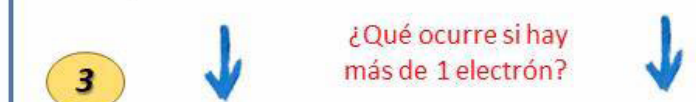

\section{Átomos polielectrónicos} Configuraciones electrónicas

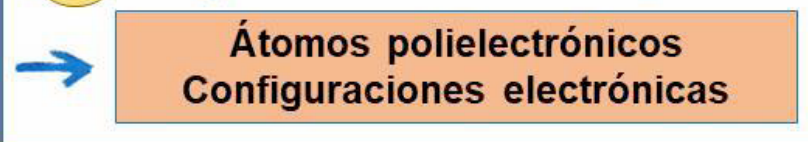

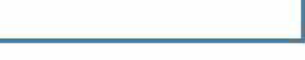

\section{¿Cómo afecta la estructura del \\ átomo y la distribución de los \\ electrones a las propiedades}

de los elementos químicos?

\section{Comprender las propiedades de los átomos de la Tabla Periódica}

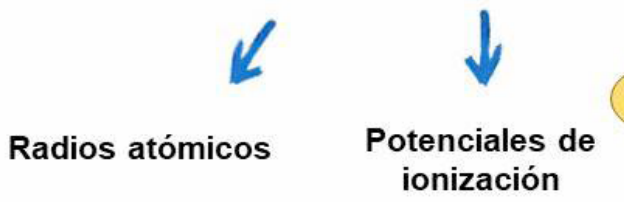

\section{4} ionización

Afinidades electrónicas

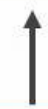

No Relacionado con la cuestión previa (№) correspondiente

Figura 1. Mapa de contenidos del tema "Estructura atómica"

Jornadas de Formación e Innovación Docente del Profesorado | № 1 (2018)

cc (i) $\Theta$ Esta obra se distribuye con la licencia Creative Commons

Reconocimiento-NoComercial-SinObraDerivada 
El mapa de contenidos muestra esencialmente los contenidos conceptuales (Petrucci, 2017) y procedimentales (estos últimos recuadrados a color), así como la jerarquización de los mismos y la conexión existente entre ellos. Los contenidos actitudinales, los cuales no se especifican en dicho mapa, se relacionan a continuación: respeto entre los alumnos, escuchar a los compañeros, capacidad crítica, comunicación oral y escrita, y capacidad de análisis y sintesis.

La experiencia docente previa muestra que la explicación del tema de forma magistral termina provocando la desconexión por parte del alumno, lo que implica que se encuentren en las semanas previas al examen con un temario extenso y complejo de entender, que terminan abandonando debido a su dificultad. Mi modelo metodológico ideal persigue la obligatoriedad de trabajar día a día los conceptos del tema, de forma que los alumnos vayan construyendo el conocimiento poco a poco. Mi modelo metodológico posible (Figura 2) consistiría en ir proponiendo una serie de preguntas concatenadas, de forma que el alumno vaya dando respuestas a las mismas y vaya construyendo "ladrillo a ladrillo" desde la base hasta el techo, los conceptos necesarios para comprender, en este caso, la estructura del átomo. El papel del profesor consistiría en plantear la búsqueda de ciertos conceptos y desarrollar las preguntas cuyas respuestas permitirán alcanzar el conocimiento del contenido y la resolución de problemas, así como estimular y motivar al estudiante para que se comprometa con esa búsqueda, y mantenga siempre un razonamiento crítico.

Jornadas de Formación e Innovación Docente del Profesorado | № 1 (2018) Esta obra se distribuye con la licencia Creative Commons 


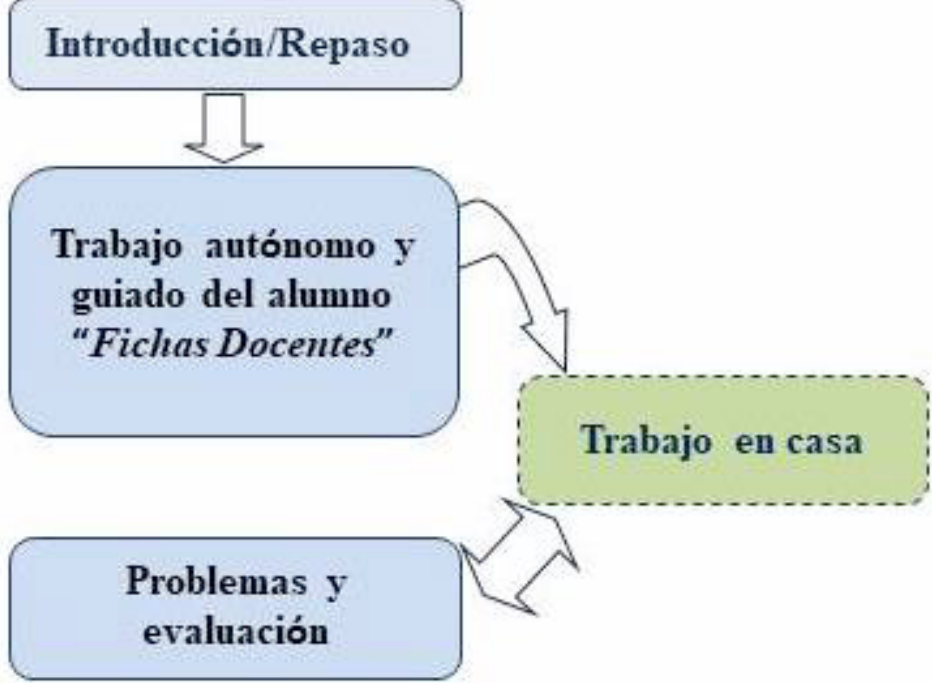

Figura 2. Modelo metodológico posible

Este modelo metodológico demanda un alto grado de compromiso, dedicación y responsabilidad por parte de los estudiantes. Se requiere de un ambiente en el que todos puedan trabajar en silencio de forma individual, adentrándose en el tema y descubriendo conceptos para seguidamente trabajar en grupo hasta conseguir comprenderlos y asimilaros, desarrollando al mismo tiempo las destrezas necesarias para la construcción del conocimiento.

La secuencia de actividades programada para este Ciclo de Mejora se presenta a continuación dividida en siete sesiones de una hora de duración cada una. Para cada sesión se ha planteado una ficha detallada que incluye una descripción de la actividad a desarrollar, la duración y los recursos necesarios para llevarla a cabo:

Jornadas de Formación e Innovación Docente del Profesorado I № 1 (2018) Esta obra se distribuye con la licencia Creative Commons 


\begin{tabular}{|l|l|c|}
\hline Actividad 1 & \multicolumn{1}{|c|}{ Descripción } & Duración \\
\hline & $\begin{array}{l}\text { Presentación de una serie de conceptos } \\
\text { básicos sobre la radiación electromag- } \\
\text { nética y ondas. Se les indica a los alum- } \\
\text { nos que amplien esta información en } \\
\text { libros e internet. }\end{array}$ & 20' \\
\hline $\begin{array}{l}\text { Se piden voluntarios para explicar } \\
\text { lo que han encontrado y lo que han } \\
\text { entiendo. }\end{array}$ & 20' \\
\hline $\begin{array}{l}\text { Presentación de una serie de conceptos } \\
\text { básicos sobre la Mecánica Cuántica. }\end{array}$ & 20' \\
\hline Recursos: PowertPoint, Internet, Libros de texto & \\
\hline
\end{tabular}

\begin{tabular}{|l|l|c|}
\hline Actividad 2 & \multicolumn{1}{|c|}{ Descripción } & Duración \\
\hline & $\begin{array}{l}\text { Se piden voluntarios para resumir y ex- } \\
\text { plicar lo entendido sobre la Mecá- } \\
\text { nica Cuántica. Se relaciona con la teoría } \\
\text { onda-corpúsculo. }\end{array}$ & \multicolumn{1}{|c|}{ 15' $^{\prime}$} \\
& $\begin{array}{l}\text { Se les propone una serie de ejercicios } \\
\text { prácticos relacionados con el tema de } \\
\text { las ondas y con el modelo atómico de } \\
\text { Bohr, donde deben aplicar la Mecánica } \\
\text { Cuántica para poder resolverlos. La pri- } \\
\text { mera mitad del tiempo les obligo a per- } \\
\text { manecer en silencio e intentar resolver } \\
\text { los problemas con ayuda de las intro- } \\
\text { ducciones y de internet. En la segunda } \\
\text { mitad le dejo debatir y preguntarse en- } \\
\text { tre ellos. Finalmente, salen a resolverlo a } \\
\text { la pizarra. }\end{array}$ & \\
\hline Recursos: PowertPoint, Internet, Pizarra \\
\hline
\end{tabular}

Jornadas de Formación e Innovación Docente del Profesorado I № 1 (2018) Esta obra se distribuye con la licencia Creative Commons Reconocimiento-NoComercial-SinObraDerivada Internacional (CC BY-NC-ND 4.0.) 


\begin{tabular}{|l|l|c|}
\hline Actividad 3 & \multicolumn{1}{|c|}{ Descripción } & Duración \\
\hline & $\begin{array}{l}\text { Explicados y adquiridos los conceptos fí- } \\
\text { sicos previos al tema, se les entrega a los } \\
\text { alumnos unas fichas de trabajo dirigido que } \\
\text { incluyen los conceptos que se pretende } \\
\text { que adquieran en la sesión. En este caso, } \\
\text { el descubrimiento del concepto de función } \\
\text { de onda y de función probabilidad (funcio- } \\
\text { nes radial y angular). La sesión se desarrolla } \\
\text { mediante un trabajo en grupos de 4-5 alum- } \\
\text { nos. Me paseo entre el alumnado para ir re- } \\
\text { solviendo dudas puntuales que les surjan y } \\
\text { le impidan seguir con el desarrollo de las fi- } \\
\text { chas, así como hacer comentarios. Al final } \\
\text { de la sesión los alumnos tendrán que tener } \\
\text { las fichas acabadas o tendrán que terminar } \\
\text { de completarlas en casa. }\end{array}$ & \\
\hline Recursos: Fichas, & Internet, Libros de texto \\
\hline
\end{tabular}

\begin{tabular}{|l|l|l|}
\hline \multicolumn{1}{|c|}{ Actividad 4 } & Descripción & Duración \\
\hline & Trabajo en casa & - \\
\hline Recursos: - & \\
\hline
\end{tabular}

\begin{tabular}{|l|l|c|}
\hline Actividad 5 & \multicolumn{1}{|c|}{ Descripción } & Duración \\
\hline & $\begin{array}{l}\text { Pido voluntarios y los alumnos harán re- } \\
\text { súmenes y resolverán las preguntas que } \\
\text { les plantee acerca de las fichas del día } \\
\text { anterior. }\end{array}$ & \multicolumn{1}{|c|}{ 15' $^{\prime}$} \\
\cline { 2 - 3 } & $\begin{array}{l}\text { Les entrego las fichas que implican el des- } \\
\text { cubrimiento de los conceptos de penetra- } \\
\text { ción y apantallamiento, orbitales atómicos } \\
\text { y números cuánticos, así como la aplica- } \\
\text { ción de las reglas de Pauli, Hund y Aufbau. } \\
\text { Me paseo entre los alumnos para resolver } \\
\text { las dudas. Al final de la sesión los alum- } \\
\text { nos tendrán que tener las fichas acabadas } \\
\text { o tendrán que terminar de completarlas } \\
\text { en casa. }\end{array}$ & 45' \\
\hline Recursos: Fichas, Internet, Pizarra, Libros de texto & \\
\hline
\end{tabular}

Jornadas de Formación e Innovación Docente del Profesorado I № 1 (2018) Esta obra se distribuye con la licencia Creative Commons Reconocimiento-NoComercial-SinObraDerivada Internacional (CC BY-NC-ND 4.0.) 


\begin{tabular}{|c|c|c|}
\hline Actividad 6 & Descripción & Duración \\
\hline & Trabajo en casa & - \\
\hline Recursos: - & \\
\hline
\end{tabular}

\begin{tabular}{|l|l|c|}
\hline Actividad 7 & \multicolumn{1}{|c|}{ Descripción } & Duración \\
\hline & $\begin{array}{l}\text { Pido voluntarios para resumir y dar las } \\
\text { claves sobre las cuestiones de las fichas } \\
\text { del día anterior. }\end{array}$ & $15^{\prime}$ \\
\hline $\begin{array}{l}\text { Les entrego las fichas en las que deberán } \\
\text { de aprender a colocar los electrones en } \\
\text { los orbitales atómicos y posteriormente } \\
\text { descubrir cómo se lleva a cabo la cons- } \\
\text { trucción de las configuraciones electró- } \\
\text { nicas. Me paseo entre los alumnos para } \\
\text { resolver las dudas. Al final de la sesión } \\
\text { los alumnos tendrán que tener las fichas } \\
\text { acabadas o tendrán que terminar de } \\
\text { completarlas en casa. }\end{array}$ & \\
\hline
\end{tabular}

Recursos: Fichas, Pizarra, Internet, Libros de texto

\begin{tabular}{|l|l|c|}
\hline \multicolumn{1}{|c|}{ Actividad 8 } & Descripción & Duración \\
\hline & Trabajo en casa & - \\
\hline Recursos: - & \\
\hline
\end{tabular}

\begin{tabular}{|l|l|c|}
\hline Actividad 9 & \multicolumn{1}{|c|}{ Descripción } & Duración \\
\hline & $\begin{array}{l}\text { Pido voluntarios y les planteo ejercicios } \\
\text { breves acerca de las cuestiones impor- } \\
\text { tantes de las fichas del día anterior, que } \\
\text { se van resolviendo simultáneamente en } \\
\text { la pizarra. }\end{array}$ & 15' \\
\hline $\begin{array}{l}\text { Les entrego las fichas en las que debe- } \\
\text { rán entender las propiedades periódicas: } \\
\text { radio atómico, potencial de ionización, } \\
\text { afinidad electrónica y electronegativi- } \\
\text { dad. Me paseo entre los alumnos para } \\
\text { resolver las dudas. Al final de la sesión } \\
\text { los alumnos tendrán que tener las fichas } \\
\text { acabadas o tendrán que terminar de } \\
\text { completarlas en casa. }\end{array}$ & \\
\hline
\end{tabular}


Recursos: Fichas, Pizarra, Internet, Libros de texto

\begin{tabular}{|l|l|c|}
\hline Actividad 10 & \multicolumn{1}{|c|}{ Descripción } & Duración \\
\hline & Trabajo en casa & - \\
\hline Recursos: - & \\
\hline
\end{tabular}

\begin{tabular}{|l|l|c|}
\hline Actividad 11 & Descripción & Duración \\
\hline $\begin{array}{l}\text { Resolución de dudas generales. } \\
\text { Cuestionario de autoevaluación de la } \\
\text { comprensión de los conceptos desarro- } \\
\text { llados en las anteriores sesiones. }\end{array}$ & $60^{\prime}$ \\
\hline Recursos: Pizarra, Programa Kahoot &
\end{tabular}

Antes de comenzar el desarrollo del tema se pasa un cuestionario de ideas previas sobre algunos de los contenidos conceptuales propuestos (cuestiones 1, 2 y 4), así como de uno de los procedimentales (cuestión 3), que será respondido de forma individual por los alumnos. Dicho test se pasará nuevamente tras la ejecución del tema, lo que permitirá observar el cambio de concepciones sobre dichos conceptos y hacer un seguimiento de la evolución de los estudiantes en relación con estos conceptos claves (Porlan, 2017). Este test consta de las siguientes cuestiones:

1. ¿Qué idea tienes de cómo es la estructura de un átomo?

2. ¿Cómo describirías lo que es un electrón?

3. ¿Por qué el orden energético de los orbitales es 1s $2 p$ 3s $3 p$ 4s $3 d 4 p 5 s ?$

4. Con tus propias palabras, ¿qué es el potencial de ionización y la afinidad electrónica?

\section{Aplicación del Ciclo de Mejora Docente}

Tras el desarrollo de las distintas actividades, se describe, a continuación, un relato resumido de las sesiones. 
En la primera sesión se explica a los alumnos que la metodología iba a cambiar, que haría un uso muy restringido del PowerPoint, que tenían que ser ellos los que fueran descubriendo los conceptos y la forma de aplicarlos a través de un trabajo guiado.La implantación de esta metodología fue ardua y trabajosa puesto que los alumnos se dedicaban más a charlar entre ellos que a buscar la información que se les pedía. En el momento de salir y explicar lo que habían encontrado, en lugar de pedir voluntarios, hice salir a los que había observado que no se dedicaron a realizar la actividad. Este momento creó un clima de tensión, que afortunadamente tuvo como recompensa que se lo tomaran con más responsabilidad y se pudo avanzar adecuadamente en los conceptos que se pretendían desarrollar sobre la radiación electromagnética y las ondas.

La segunda sesión comenzó de forma muy distinta, los alumnos estaban todos muy comprometidos, callados, trabajadores y participativos cuando se requería... Esto me permitió llevar a cabo debates de resolución de problemas, en los que alumnos exponían sus dudas y dificultades sobre el modelo atómico de Bohr y el uso de la Mecánica Cuántica a la hora de resolver los ejercicios. Seguidamente, otros compañeros explicaron con sus palabras como lo veían y que procedimiento seguían, "las visiones y trucos" de cada uno se indicaban en la pizarra. Todo ello fue muy fructífero puesto que facilitó el entendimiento de los alumnos de estos temas tan arduos.

Tras el desarrollo de unos conocimientos básicos previos, en la tercera sesión comenzaba lo fundamental del tema. Para esta parte se recurrió al sistema metodológico indicado previamente, la entrega a los alumnos de unas fichas de trabajo dirigido, que incluyen los conceptos que se pretende que adquieran en la sesión. La sesión se desarrolló mediante trabajo en grupos de 4-5 alumnos. Durante su trabajo, me paseé entre el alumnado para ir resolviendo las dudas puntuales que les surgían y le impedían seguir con el desarrollo de las fichas, así como para hacerles 
comentarios. Al finalizar la sesión, algunos alumnos terminaron la ficha y otros no. Por ello, pedí que la terminaran en casa para poder seguir la clase del día siguiente.

La cuarta sesión comenzó con un coloquio acerca de los conceptos del día anterior. La clase fluyó satisfactoriamente puesto que la gran mayoría de los alumnos había trabajado el tema, traían afianzados muchos conceptos, lo que les permitía debatir y criticar los comentarios y resoluciones de los demás. En esta parte del temario aparecieron algunos conceptos y gráficas complicadas. Para su comprensión puse ejemplos, de los cuales fuimos sacando conclusiones y así pudieron llegar a comprenderlos. Fue una clase muy productiva, puesto que su trabajo previo con la ficha les permitía saber de lo que se estaba hablando, entender lo que se estaba explicando, así como hacer comentarios críticos y constructivos del tema.

Esta dinámica de trabajo fue la que se siguió y se mantuvo durante la quinta y sexta sesión del tema. Durante ellas se ha intentó acentuar la participación de todos los alumnos, haciéndoles ver que toda opinión es útil y constructiva. Finalmente, en una séptima sesión se hizo un cuestionario de autoevaluación para comprobar la comprensión de los conceptos desarrollados en estas sesiones. Para ello, se usó el programa Kahoot, el cual permite realizar una evaluación de forma menos agresiva, siendo incluso ameno y divertido para los estudiantes. Este test me confirmó que los alumnos se estaban enterando, me dio ánimos para continuar con este sistema y esperanzas para mejorar los desastrosos resultados de años anteriores.

La sensación, tras estas sesiones, se podría resumir con varias palabras: aceptación, entusiasmo, interés y conocimiento. El gran inconveniente de este sistema metodológico basado en el trabajo del alumno y en el descubrimiento autónomo de muchos conceptos es el ralentizamiento de las clases y con ello en el avance de la asignatura. Esto me está obligando a plantearme algunos 
cambios, a seleccionar y priorizar los contenidos y a rediseñar las próximas fichas en ese camino guiado del aprendizaje.

Para llevar a cabo una evaluación del aprendizaje de los estudiantes se ha analizado el cuestionario previo y final propuesto anteriormente. El cuestionario, constituido de cuatro preguntas, se pasó al comienzo de la primera sesión (test inicial) y al final de la séptima sesión (test final). A continuación, se presentan las escaleras de aprendizaje para cada una de esas preguntas (Figura 3):

Pregunta 1

\section{Pregunta 2}

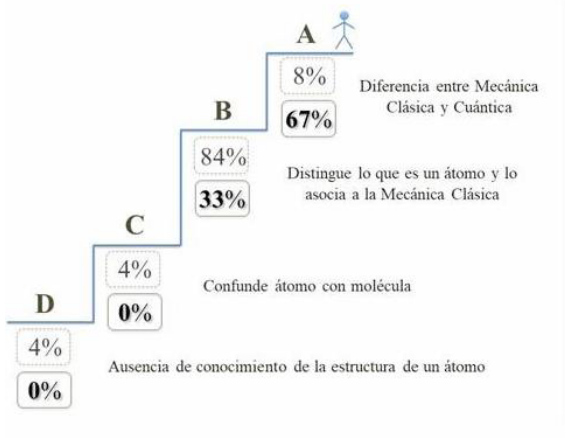

Pregunta 3

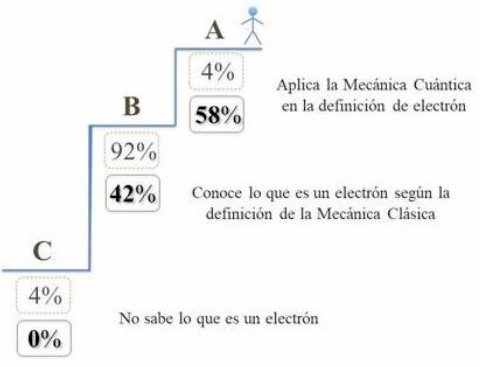

Pregunta 4

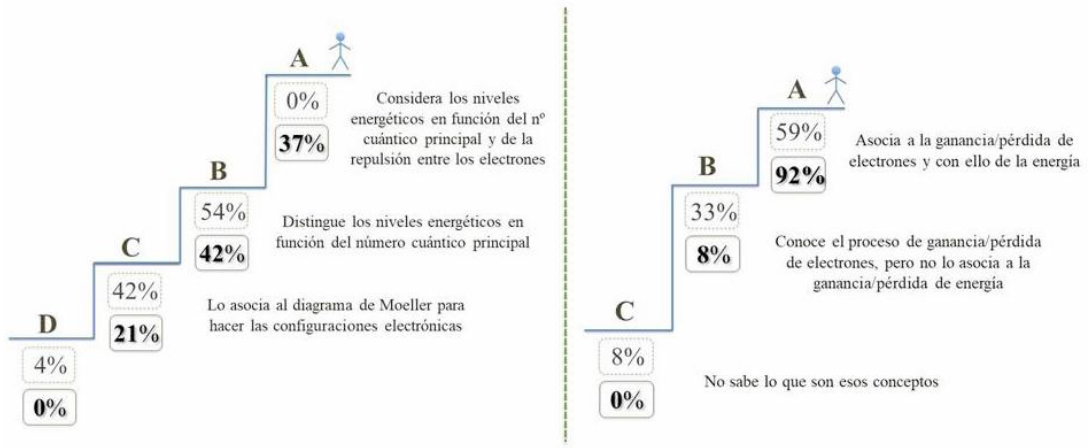

Figura 3. Escaleras de aprendizaje

En las anteriores escaleras de aprendizaje se han ido definiendo distintos niveles de progresión en cuanto a la

Jornadas de Formación e Innovación Docente del Profesorado I № 1 (2018)
Esta obra se distribuye con la licencia Creative Commons 
concepción (test inicial) o adquisición (test final) de los contenidos. Para todas las preguntas, el nivel base se corresponde con una ausencia del conocimiento. A partir de él, se distinguen distintos niveles en función de la veracidad o complejidad en la comprensión del concepto, hasta llegar al nivel superior donde el alumno demuestra una noción totalmente integrada del mismo. En ellas, además, se indica el porcentaje de estudiantes que se encuentran en cada nivel de aprendizaje, recuadrándolo con líneas discontinuas los resultados correspondientes al test inicial y con línea continua (y en negrita) los del test final.

La progresión de los alumnos, tras haberse desarrollado el tema, se puede observar en la siguiente tabla:

Tabla 1. Progresión de cada alumno desde el cuestionario inicial al cuestionario final

\begin{tabular}{|c|c|c|c|c|c|c|c|c|c|c|c|c|}
\hline \multirow[b]{3}{*}{ Sujeto 1} & \multicolumn{6}{|c|}{ Niveles de desarrollo iniciales y finales } & \multicolumn{6}{|c|}{ Niveles de desarrollo iniciales y finales } \\
\hline & \multicolumn{3}{|c|}{ Pregunta 1} & \multicolumn{3}{|c|}{ Pregunta 2} & \multicolumn{3}{|c|}{ Pregunta 3} & \multicolumn{3}{|c|}{ Pregunta 4} \\
\hline & B & A & 4 & B & A & $A$ & B & A & 1 & A & A & $\leftrightarrow$ \\
\hline Sujeto 2 & B & A & $A$ & B & A & 1 & B & A & 1 & A & A & $\leftrightarrow$ \\
\hline Sujeto 3 & B & A & 4 & B & A & 1 & c & B & $A$ & A & A & $\rightarrow$ \\
\hline Sujeto 4 & B & A & 4 & B & A & $A$ & c & A & $A \wedge$ & A & A & $\rightarrow$ \\
\hline Sujeto 5 & B & A & $A$ & B & A & $A$ & c & B & 4 & B & A & $A$ \\
\hline Sujeto 6 & c & B & 4 & c & B & A & D & C & 4 & C & B & 1 \\
\hline Sujeto 7 & B & B & $\leftrightarrow$ & B & A & 1 & c & B & $A$ & A & A & $\Leftrightarrow$ \\
\hline Sujeto 8 & A & A & $\Leftrightarrow$ & B & A & 1 & B & B & $\leftrightarrow$ & B & A & 1 \\
\hline Sujeto 9 & B & B & $\rightarrow$ & B & B & $\rightarrow$ & c & C & $\rightarrow$ & c & A & $A$ \\
\hline Sujeto 10 & B & A & 1 & B & A & $A$ & B & A & $A$ & A & A & $\leftrightarrow$ \\
\hline Sujeto 11 & B & A & $A$ & B & A & $A$ & c & B & $A$ & A & A & $\leftrightarrow$ \\
\hline Sujeto 12 & B & A & 4 & B & A & 1 & B & A & 1 & A & A & $\leftrightarrow$ \\
\hline Sujeto 13 & B & B & 1 & B & B & $\leftrightarrow$ & c & A & AA & A & A & $\leftrightarrow$ \\
\hline Sujeto 14 & A & A & $\Leftrightarrow$ & B & A & 4 & B & B & $\leftrightarrow$ & B & A & 1 \\
\hline Sujeto 15 & D & B & A & B & B & $\leftrightarrow$ & B & B & $\leftrightarrow$ & A & A & $\leftrightarrow$ \\
\hline Sujeto 16 & B & B & $\leftrightarrow$ & B & B & $\leftrightarrow$ & c & A & AA & A & A & $\leftrightarrow$ \\
\hline Sujeto 17 & B & A & $A$ & B & A & $A$ & c & C & $\leftrightarrow$ & B & B & $\cdots$ \\
\hline Sujeto 18 & B & B & $\Leftrightarrow$ & B & B & $\leftrightarrow$ & c & C & $\rightarrow$ & B & A & $A$ \\
\hline Sujeto 19 & B & A & $A$ & A & A & $\leftrightarrow$ & c & B & A & B & A & $A$ \\
\hline Sujeto 20 & B & A & 1 & B & B & $\leftrightarrow$ & B & A & $A$ & A & A & $\leftrightarrow$ \\
\hline Sujeto 21 & B & B & $\Leftrightarrow$ & B & B & $\Leftrightarrow$ & B & B & $\leftrightarrow$ & A & A & $\leftrightarrow$ \\
\hline Sujeto 22 & B & A & 1 & B & A & 1 & C & B & A & B & A & 1 \\
\hline Sujeto 23 & B & A & 1 & B & B & $\Leftrightarrow$ & c & C & $\Leftrightarrow$ & B & A & A \\
\hline Sujeto 24 & B & A & 1 & B & B & $\Leftrightarrow$ & B & A & 1 & A & A & $\leftrightarrow$ \\
\hline
\end{tabular}

Analizando los resultados de estos cuestionarios se pone de manifiesto una clara evolución de la mayoría de los estudiantes en la adquisición de los contenidos de este tema, puesto que prácticamente todos han ido subiendo de nivel de conocimiento. En la tercera columna de cada apartado se muestra gráficamente (con una flecha) la evolución de cada sujeto, observándose muchas flechas de estanqueidad, pero en su gran mayoría se encuentran 
en el nivel más alto de la escalera. Por otro lado, cabe destacar que la mayoría de los alumnos presentan un conocimiento alto o muy alto en la concepción de los contenidos desarrollados.

\section{Evaluación del Ciclo de Mejora Docente puesto en práctica}

Una vez finalizado el ciclo de mejora docente y tras realizar una evaluación global del mismo, puedo decir que esta experiencia docente ha resultado muy positiva.

En muchas ocasiones, los docentes nos centramos en desarrollar un temario sin interactuar con los alumnos, sin conocer su situación de partida y sin observarlos a lo largo del proceso de enseñanza-aprendizaje. Este curso me ha permitido darme cuenta de la importancia de esta interacción mutua, desde el primer día de clase hasta la evaluación. Es fundamental conocer el conocimiento base del que partimos, el tipo de audiencia que tenemos, la forma y velocidad de impartición de los contenidos, así como la evaluación de este proceso de enseñanza-aprendizaje (Bain, 2005). En cuanto a los contenidos, éstos siempre suelen venir impuestos por la tradición o por otros compañeros. Sin embargo, el ciclo de mejora me ha inducido a reflexionar acerca de los mismos, a llevar a cabo una tarea de selección previa, a ser capaz de priorizar la impartición de unos sobre otros y a interconectar ideas. Para todo ello resulta muy útil y práctica la elaboración de un mapa de contenidos, el cual nos permite ver con facilidad y de forma sintética el diseño elaborado sobre el tema y la ordenación de las distintas actividades docentes a realizar en él.

En referencia al proceso de evaluación, me parece muy útil hacer un test de ideas previas y otro de ideas finales tras la impartición de un tema de la asignatura. Por un lado, el test inicial nos permite conocer el punto de partida de los alumnos, y con ello saber a qué tipo de contenidos 
dedicarle menos tiempo y en cuales profundizar más. Por otro lado, el test final nos permite analizar si el modelo docente empleado ha sido exitoso o no. La consideración simultanea de ambos test se plasma en las denominadas escaleras de aprendizaje, las cuales se convierten en una herramienta excelente para conocer si la transmisión del conocimiento está fluyendo adecuadamente y si el diseño del mapa de contenido y la metodología puesta en juego están siendo útiles para los alumnos.

Para cursos venideros me gustaría continuar con la misma metodología basada en "fichas de aprendizaje guiado" con las que los alumnos puedan ir construyendo su propio aprendizaje, y acompañarlas de debates con los que se consigan resolver dudas generales y permitan captar los errores generales. La aceptación de esta metodología por parte de los estudiantes ha sido muy buena, manifestándose en su gran implicación en las distintas actividades, y en los resultados obtenidos, los cuales han sido muy productivos. Por todo ello, incorporaré esta metodología a mi práctica docente habitual, manteniéndola en constante proceso de evaluación. En base a esto, se han detectado aspectos a mejorar en dichas fichas, requiriéndose correcciones de las mismas y una distribución mejorada en el tiempo. Para ello, pretendo emplear más meticulosamente los test iniciales ya que me pueden aportar un análisis más real del punto de partida de los estudiantes y esto a su vez poder ajustar mejor el mapa de contenidos y consecuentemente el diseño de las secuencias de actividades. 


\section{Referencias bibliográficas}

1) Finkel, D. (2008). Dar clase con la boca cerrada. Valencia: Publicaciones Universitat de Valencia.

2) Petrucci, R. H.; Herring, F. G.; Madura, J. D.; Bissonnette, C. (2017). Química General. Principios y aplicaciones modernas. Madrid: Pearson.

3) Porlan, R. Coord. (2017). Enseñanza universitaria. Cómo mejorarla. Madrid: Morata.

4) Bain, K. (2005). Lo que hacen los mejores profesores de universidad. Valencia: Publicaciones Universitat de Valencia. 